\title{
INTERSTELLAR METASTABLE HELIUM ABSORPTION AS A PROBE OF THE COSMIC-RAY IONIZATION RATE
}

\author{
Nick Indriolo ${ }^{1}$, L. M. Hobbs ${ }^{2}$, K. H. Hinkle ${ }^{3}$, and Benjamin J. McCall ${ }^{1,4}$ \\ ${ }^{1}$ Department of Astronomy, University of Illinois at Urbana-Champaign, Urbana, IL 61801, USA \\ ${ }^{2}$ University of Chicago, Yerkes Observatory, Williams Bay, WI 53191, USA \\ ${ }^{3}$ National Optical Astronomy Observatories, Tucson, AZ 85726, USA \\ ${ }^{4}$ Department of Chemistry, University of Illinois at Urbana-Champaign, Urbana, IL 61801, USA \\ Received 2009 February 25; accepted 2009 August 24; published 2009 September 16
}

\begin{abstract}
The ionization rate of interstellar material by cosmic rays has been a major source of controversy, with different estimates varying by three orders of magnitude. Observational constraints of this rate have all depended on analyzing the chemistry of various molecules that are produced following cosmic-ray ionization, and in many cases these analyses contain significant uncertainties. Even in the simplest case $\left(\mathrm{H}_{3}^{+}\right)$, the derived ionization rate depends on an (uncertain) estimate of the absorption path length. In this paper, we examine the feasibility of inferring the cosmic-ray ionization rate using the $10830 \AA$ absorption line of metastable helium. Observations through the diffuse clouds toward HD 183143 are presented, but yield only an upper limit on the metastable helium column density. A thorough investigation of $\mathrm{He}^{+}$chemistry reveals that only a small fraction of $\mathrm{He}^{+}$will recombine into the triplet state and populate the metastable level. In addition, excitation to the triplet manifold of helium by secondary electrons must be accounted for as it is the dominant mechanism which produces He* in some environments. Incorporating these various formation and destruction pathways, we derive new equations for the steady state abundance of metastable helium. Using these equations in concert with our observations, we find $\zeta_{\mathrm{He}}<1.2 \times 10^{-15} \mathrm{~s}^{-1}$, an upper limit about 5 times larger than the ionization rate previously inferred for this sight line using $\mathrm{H}_{3}^{+}$. While observations of interstellar $\mathrm{He}^{*}$ are extremely difficult at present, and the background chemistry is not nearly as simple as previously thought, potential future observations of metastable helium would provide an independent check on the cosmic-ray ionization rate derived from $\mathrm{H}_{3}^{+}$in diffuse molecular clouds, and, perhaps more importantly, allow the first direct measurements of the ionization rate in diffuse atomic clouds.
\end{abstract}

Key words: astrochemistry - atomic processes - cosmic rays

\section{INTRODUCTION}

\subsection{Motivation}

Over the past several decades, the assumed value of the cosmic-ray ionization rate of interstellar hydrogen has fluctuated up and down. Various theories and models have predicted ionization rates from $10^{-18} \mathrm{~s}^{-1}$ to $10^{-15} \mathrm{~s}^{-1}$ in the diffuse interstellar medium (e.g. Spitzer \& Tomasko 1968; van Dishoeck \& Black 1986; Webber 1998; Le Petit et al. 2004). On the other hand, observations of molecules such as HD and OH typically resulted in estimates of the ionization rate that were on the order of $10^{-17} \mathrm{~s}^{-1}$ (O'Donnell \& Watson 1974; Black \& Dalgarno 1977; Black et al. 1978; Hartquist et al. 1978a, 1978b; Federman et al. 1996). However, these estimates depend on gas phase abundances of $\mathrm{O}, \mathrm{OH}, \mathrm{D}$, and $\mathrm{HD}$, values which are often difficult to measure precisely. More recently, observations of $\mathrm{H}_{3}^{+}$ have again revised the cosmic-ray ionization rate upward to a few times $10^{-16} \mathrm{~s}^{-1}$ (McCall et al. 2003; Indriolo et al. 2007). Deriving the ionization rate from $\mathrm{H}_{3}^{+}$requires only one uncertain parameter, the absorption path length. While the higher ionization rates derived from $\mathrm{H}_{3}^{+}$are becoming generally accepted (Dalgarno 2006), it is desirable to search for new observables which can offer independent and less uncertain estimates of the ionization rate.

In this paper, we investigate the possibility of observationally determining the total ionization rate of helium atoms by cosmic rays in diffuse clouds. The basic premise is that in a sufficiently reddened cloud, the column density of neutral helium atoms excited to the metastable $1 \mathrm{~s} 2 \mathrm{~s}{ }^{3} \mathrm{~S}_{1}$ level may be high enough to be measured by means of interstellar absorption lines arising from that level. The high cosmic abundance of helium and the long radiative lifetime of the metastable level, $A^{-1}=2.5 \mathrm{hr}$, may compensate for the difficulty of populating this highly excited level, which lies $19.8 \mathrm{eV}$ above the ground level. Previously, it has been assumed that this level should be populated almost entirely by cosmic-ray ionization of helium atoms, followed by radiative recombination of the ions with electrons. Figure 1 schematically shows the processes conventionally used in describing the (de)population of the metastable state.

\subsection{Background}

A simple reaction network-consisting of (1) cosmic-ray ionization of $\mathrm{He}^{0}$ atoms in the $1 \mathrm{~s}^{2}{ }^{1} \mathrm{~S}_{0}$ ground level, (2) radiative recombination of $\mathrm{He}^{+}$ions with electrons to the metastable $1 \mathrm{~s} 2 \mathrm{~s}$ ${ }^{3} \mathrm{~S}_{1}$ level, and (3) forbidden spontaneous emission to the ground level—was first used by Scherb (1968) and Rees et al. (1968) in proposing the observability of interstellar metastable helium. However, both of these studies considered two-photon emission from the metastable state, then thought to be the dominant method of depopulation. This two-photon decay proceeds on a timescale of $A^{-1} \gtrsim 116$ days (Drake \& Dalgarno 1968). In the following year, the radiative lifetime associated with one-photon decay was computed to be $A^{-1} \approx 7 \mathrm{hr}$ (Griem 1969), nearly 400 times faster than the two-photon decay. This value was later refined to $A^{-1}=2.5 \mathrm{hr}$ (Woodworth \& Moos 1975; Hata \& Grant 1981), still much faster, and as a result the analyses performed by Scherb (1968) and Rees et al. (1968) had greatly overestimated the population in the metastable state (this possibility was noted by Rees et al. 1968). Consequently, 


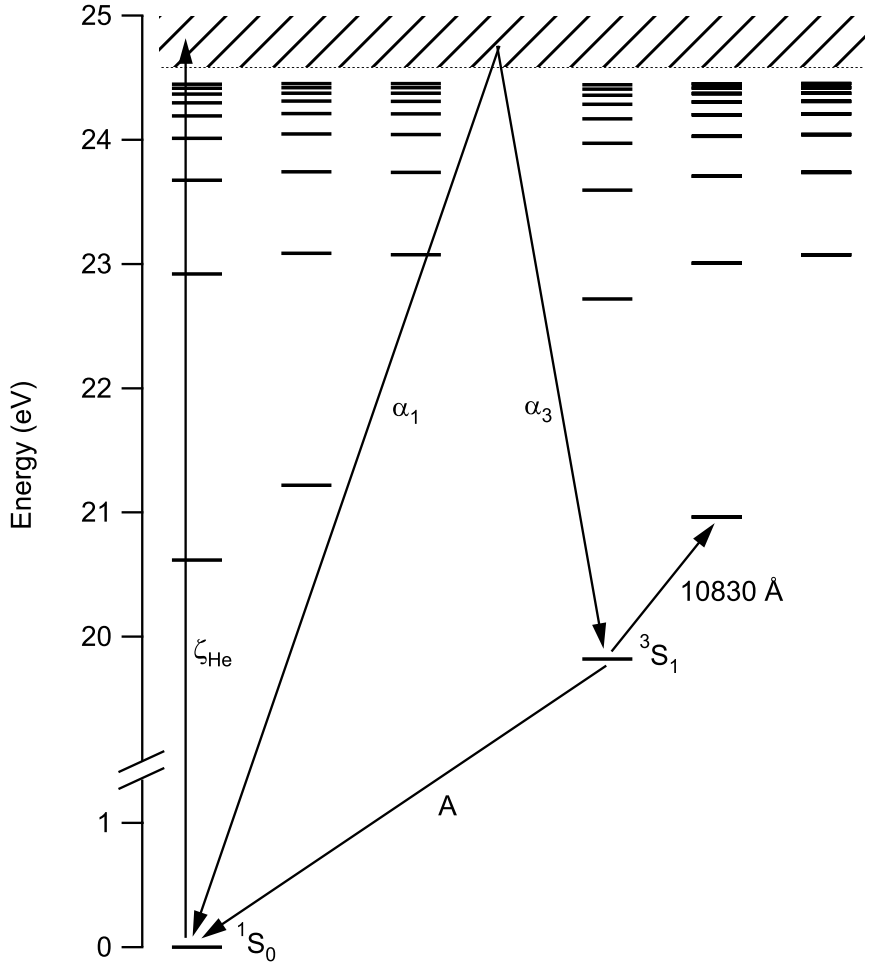

Figure 1. Simplified energy level diagram of atomic helium, showing only S, P, and $\mathrm{D}$ terms up through a principal quantum number of 10 . The key processes thought to control the abundance of the metastable $2{ }^{3} \mathrm{~S}_{1}$ state, along with the absorption line from this state at $10830 \AA$, are indicated. It is assumed that all electron recombinations into either the singlet or triplet manifold quickly lead to the ground state of that manifold by allowed spontaneous emission.

the thought of observing interstellar metastable helium was abandoned.

Because of the high ionization rate inferred from $\mathrm{H}_{3}^{+}$, we decided to revisit these calculations considering up-to-date rate coefficients and improved telescope/detector capabilities. Assuming the same chemical scheme as in the past, we can derive the steady state equations for the ground, ionized, and metastable states:

$$
\begin{gathered}
\alpha_{1} n_{e} n_{i}+n_{m} A=n_{g} \zeta_{\mathrm{He}}, \\
n_{g} \zeta_{\mathrm{He}}=\left(\alpha_{1}+\alpha_{3}\right) n_{e} n_{i}, \\
\alpha_{3} n_{e} n_{i}=n_{m} A .
\end{gathered}
$$

Here, $n_{g}, n_{i}$, and $n_{m}$ denote the populations of the ground, ionized, and metastable levels, respectively; $\zeta_{\mathrm{He}}$ is the total ionization rate of $\mathrm{He}^{0}$ atoms due to cosmic rays, including the effects of secondary electrons; $\alpha_{1}$ and $\alpha_{3}$ are the total direct recombination rates to all singlet levels and to all triplet levels, respectively; $n_{e}$ is the electron density in the gas; and $A$ is the Einstein coefficient for spontaneous emission from the metastable level. The units of each term in Equations (1)(3) are $\mathrm{cm}^{-3} \mathrm{~s}^{-1}$. All recombinations to triplet levels above the metastable level are assumed to produce subsequent radiative cascades to the metastable level that are effectively instantaneous, owing to the long lifetime of the latter level. Similarly, all recombinations to singlet levels are assumed to cascade promptly to the ground level. Equations (2) and (3) can be solved for the ratios $n_{i} / n_{g}$ and $n_{m} / n_{i}$, and thus for $n_{m} / n_{g}$ as well. These ratios can be substituted into the definition of the fractional population of the metastable level,

$$
f_{m}=\frac{n_{m}}{\left(n_{m}+n_{i}+n_{g}\right)}
$$

in order to obtain the desired relation between the fractional metastable population $f_{m}$ and the ionization rate $\zeta_{\mathrm{He}}$,

$$
\frac{1}{f_{m}}=1+\frac{A}{\left(\alpha_{3} n_{e}\right)}+\frac{A}{\left(b \zeta_{\mathrm{He}}\right)}
$$

The triplet branching fraction for recombinations at $70 \mathrm{~K}$ is $b=\alpha_{3} /\left(\alpha_{1}+\alpha_{3}\right)=0.62$, since $\alpha_{1}=4.0 \times 10^{-12} \mathrm{~cm}^{3}$ $\mathrm{s}^{-1}$ and $\alpha_{3}=6.6 \times 10^{-12} \mathrm{~cm}^{3} \mathrm{~s}^{-1}$ (R. Porter 2009, private communication). Radiative decay to the ground level is by far the fastest of the three processes mentioned above, with $A=$ $1.1 \times 10^{-4} \mathrm{~s}^{-1}$. In contrast, $n_{e}=0.02 \mathrm{~cm}^{-3}, \alpha_{3} n_{e}=1.3 \times 10^{-13}$ $\mathrm{s}^{-1}$, and $\zeta_{\mathrm{He}}=3 \times 10^{-16} \mathrm{~s}^{-1}$ are representative values in diffuse clouds. Given these values, $1 \ll A /\left(\alpha_{3} n_{e}\right) \ll A /\left(b \zeta_{\mathrm{He}}\right)$, and Equation (5) can be approximated by

$$
\frac{1}{f_{m}} \approx \frac{A}{b \zeta_{\mathrm{He}}} .
$$

Owing to the very large differences among the rates, this approximation to Equation (5) is nearly exact. This holds true as long as $\zeta_{\mathrm{He}} \ll n_{e}\left(\alpha_{1}+\alpha_{3}\right) \sim 10^{-13} \mathrm{~s}^{-1}$, such that ionization of helium by cosmic rays is the rate-limiting step in the path to the metastable state. In this limit, $f_{m}$ effectively depends on $\zeta_{\mathrm{He}}$ alone-apart from the well-determined atomic constants $b$ and $A$-thus suggesting metastable helium as a fairly robust indicator of the cosmic-ray ionization rate.

\section{OBSERVATIONS}

\subsection{Predictions}

The fundamental question remaining then is whether the interstellar lines of $\mathrm{He}^{*}$ arising from a suitable diffuse cloud are likely to be detectable. The strengths of these lines are fixed by the cloud's column density of metastable atoms, $N_{m}$, which can be calculated from

$$
N_{m}=f_{m} N(\mathrm{He})=f_{m} A(\mathrm{He}) N_{\mathrm{H}},
$$

where $N(\mathrm{He})$ is the total column density of helium atoms in all states, $N_{\mathrm{H}}$ is the total column density of hydrogen nuclei $\left[N_{\mathrm{H}}=N(\mathrm{H})+2 N\left(\mathrm{H}_{2}\right)\right]$, and $A(\mathrm{He})=N(\mathrm{He}) / N_{\mathrm{H}}=0.097$ is the relative abundance of helium with respect to hydrogen (Anders \& Grevesse 1989). The fraction of interstellar helium sequestered in the grains has also been assumed negligible. If a direct measurement of $N_{\mathrm{H}}$ is not available, an alternative is to use $N_{\mathrm{H}}=\beta E(B-V)$, where $E(B-V)$ is the observed color excess, and $\beta=N_{\mathrm{H}} / E(B-V)=5.8 \times 10^{21} \mathrm{~cm}^{-2} \mathrm{mag}^{-1}$ is the interstellar gas-to-dust ratio (Bohlin et al. 1978).

To estimate the expected line strengths, we assume $E(B-$ $V)=1.0 \mathrm{mag}$ and $\zeta_{\mathrm{He}}=3 \times 10^{-16} \mathrm{~s}^{-1}$ in a suitable, individual interstellar cloud. The former value leads to $N_{\mathrm{H}}=5.8 \times$ $10^{21} \mathrm{~cm}^{-2}$ and $N(\mathrm{He})=5.6 \times 10^{20} \mathrm{~cm}^{-2}$. A substitution of the assumed value of $\zeta_{\mathrm{He}}$ into Equation (6) gives $f_{m}=1.7 \times 10^{-12}$. Then, $N_{m}=f_{m} N(\mathrm{He})=9.5 \times 10^{8} \mathrm{~cm}^{-2}$. The best choice among the available $\mathrm{He} \mathrm{I}^{*}$ lines is the $1 \mathrm{~s} 2 \mathrm{~s}{ }^{3} \mathrm{~S}-1 \mathrm{~s} 2 \mathrm{p}{ }^{3} \mathrm{P}$ multiplet located near $10830 \AA$. Data for the transitions associated with this multiplet are shown in Table 1, where column 4 gives the oscillator strengths. These lines are stronger than 
Table 1

The $1 \mathrm{~s} 2 \mathrm{~s}{ }^{3} \mathrm{~S}-1 \mathrm{~s} 2 \mathrm{p}{ }^{3} \mathrm{P}$ Multiplet of He I

\begin{tabular}{cccc}
\hline \hline$\lambda_{\text {air }}(\AA)$ & $J($ Lower $)$ & $J($ Upper $)$ & $f$ \\
\hline 10829.0911 & 1 & 0 & 0.060 \\
10830.2501 & 1 & 1 & 0.180 \\
10830.3398 & 1 & 2 & 0.300 \\
\hline
\end{tabular}

Note. Wavelengths and oscillator strengths are from the NIST Atomic Spectra Database (Ralchenko et al. 2008).

other transitions arising from the metastable level (such as the multiplet near $3889 \AA$ ), and the near-infrared wavelength is advantageous in observations of heavily reddened stars with large total column densities of helium.

Assuming $N_{m}=9.5 \times 10^{8} \mathrm{~cm}^{-2}$, the equivalent width of an unresolved blend of the two strongest lines of the multiplet, which are separated by only $2.5 \mathrm{~km} \mathrm{~s}^{-1}$, would be $W_{\lambda}=0.47 \mathrm{m \AA}$. If a spectrometer with a resolving power of 70,000 were used, the line would have a central depth of $\sim 0.30 \%$, thus demanding a signal-to-noise ratio $(\mathrm{S} / \mathrm{N})$ of $\sim 1000$ on the continuum for a $3 \sigma$ detection. Modern optical echelle spectrographs can easily reach $\mathrm{S} / \mathrm{N}$ exceeding 2000 (e.g., Ádámkovics et al. 2003), but reaching such a high S/N in the near-infrared is a significant challenge.

\subsection{Target Selection}

In choosing a target, we searched for sight lines that had a combination of several desirable characteristics: high color excess, high cosmic-ray ionization rate as inferred from $\mathrm{H}_{3}^{+}$, relatively bright $J$-band magnitude, few interstellar velocity components, and well-behaved stellar absorption features. Using these criteria, we arrived at HD 183143 as our most favorable target, with $J=4.18, V=6.86, E(B-V)=1.27$, and a spectral type of B7Iae. The star's photospheric He I absorption lines at 5875,6678 , and $7065 \AA$ are relatively broad, with FWHM $\geqslant$ $66 \mathrm{~km} \mathrm{~s}^{-1}$, and they are also free from any interfering emission components (Thorburn et al. 2003). This suggests that the photospheric $10830 \AA$ A line may desirably provide a smooth background with a shallow slope, against which one could search for the much narrower, weak interstellar lines. In addition, many interstellar absorption lines along this line of sight have been previously studied, including $\mathrm{CH}$ and $\mathrm{CH}^{+}$(Gredel et al. 1993), and $\mathrm{CN}, \mathrm{H}_{3}^{+}$, and ${ }^{12} \mathrm{CO}$ (McCall et al. 2002). These observations reveal that there are two distinct interstellar cloud groups at different velocities. Although this means that not all of the interstellar helium is at one velocity, it does provide a very useful method for potentially confirming a detection. Additionally, $\mathrm{H}_{3}^{+}$ observations have been used to determine the ionization rate of molecular hydrogen, $\zeta_{2}$, in this sight line (Indriolo et al. 2007).

Using values specific to the HD 183143 sight line $(E(B-$ $V)=1.27 ; \zeta_{\mathrm{He}}=3.5 \times 10^{-16} \mathrm{~s}^{-1}$ ), we can again perform the calculations in Section 2.1 to determine the expected line strength. The resulting equivalent width is $W_{\lambda}=0.70 \mathrm{~m} \AA$. Because the sight line has two velocity components though, we assume equal amounts of material in each cloud, and thus expect two absorption lines with $W_{\lambda}=0.35 \mathrm{~m} \AA$. These would require $\mathrm{S} / \mathrm{N} \sim 1300$ for a $3 \sigma$ detection given the same instrument capabilities assumed above. While obtaining a $\mathrm{S} / \mathrm{N}$ this high is difficult in the near-infrared, some of the most advanced telescope/detector combinations are capable of approaching such results, so we proceeded with observations.

\subsection{Execution}

Data were taken near the He I* line at $10830 \AA$ using the Phoenix spectrometer (Hinkle et al. 2002) on the Gemini South Telescope. The spectrometer was used with its echelle grating and 0.17 slit to produce a resolving power of $\sim 70,000$, and the J9232 filter to select the correct order. Observations of both the target (HD 183143) and standard ( $\alpha$ Aql) stars were taken on 2008 May 25 and 2008 June 28. Total integration times were 33 and 30 minutes for the target and 1.9 and 1.4 minutes for the standard on each night, respectively. During each set of observations, the star was nodded along the slit in an ABBA pattern to allow for the later subtraction of neighboring images, and thus the removal of the atmospheric background and detector bias levels.

\section{DATA REDUCTION}

A significant portion of the data reduction-dark current subtraction, subtraction of neighboring images, removal of bad pixels, flat fielding, combination of exposures with the spectral image in the same nod position, fitting of the spectral response, and spectral extraction-was performed using NOAO's IRAF package $^{5}$. During this process, we combined the methods outlined by Kulesa (2002) with those given by NOAO's online Phoenix documentation ${ }^{6}$ in order to obtain the best possible $\mathrm{S} / \mathrm{N}$. Once the one-dimensional spectra were extracted, they were imported to IGOR Pro ${ }^{7}$, where we have macros set up to complete the reduction (McCall 2001).

Because of the annual shift in the relative positions of (inter)stellar and atmospheric features with time, the data from different nights were first analyzed separately. In all cases, however, the expected locations of the interstellar He I* lines lie within the broad stellar absorption line. Because the $\mathrm{S} / \mathrm{N}$ of the standard star was actually lower than that of the target in the June data, we decided to forego the process of ratioing by the standard star, and instead directly fit the stellar absorption feature. The A and B beams for each night were wavelengthcalibrated using atmospheric lines and then added together. The broad stellar absorption feature was then fit using the summation of three Gaussian functions, all of which were constrained to have a full width at half-maximum (FWHM) at least 3 times that of the $10 \mathrm{~km} \mathrm{~s}^{-1}$ measured for interstellar absorption features along the line of sight. The spectra from each night were then divided by their respective fits and shifted to be in the local standard of rest (LSR) frame. Finally, the fully reduced spectra from both nights were added together and converted to velocity space to produce the top spectrum shown in Figure 2.

\section{RESULTS}

There is no indication of interstellar He* absorption at either of the expected velocities. While we did obtain a relatively high $\mathrm{S} / \mathrm{N}(\sim 700)$ for high-resolution infrared spectroscopy, we were unable to achieve the desired $\mathrm{S} / \mathrm{N} \sim 1300$. The non-detection of the He I* lines enabled us to calculate an upper limit to the column density of metastable helium along this line of sight. First, the upper limit to the equivalent width, $W_{\lambda}$, was computed via

$$
W_{\lambda}<\sigma \lambda_{\text {pix }} \sqrt{\mathcal{N}_{\text {pix }}}
$$

\footnotetext{
5 See http://iraf.noao.edu/.

6 See http://www.noao.edu/usgp/phoenix/phoenix.html.

7 See http://www.wavemetrics.com/.
} 


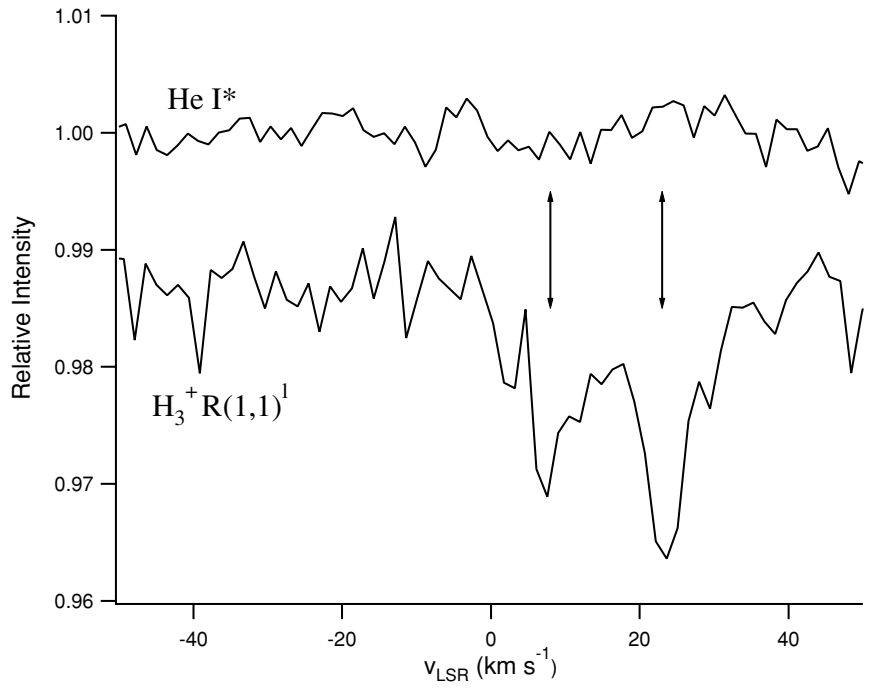

Figure 2. Spectra of HD 183143 in velocity space. The top spectrum, observed with Phoenix at Gemini South, has been adjusted for the centroid of the unresolved blend of the 1-1 and 1-2 members of the He I* multiplet, and the broad photospheric line has been divided out. The bottom spectrum (from McCall et al. 2002) shows the $R(1,1)^{l}$ transition of $\mathrm{H}_{3}^{+}$for reference. Arrows indicate the two interstellar velocity components which have been observed in various molecules $\left(\mathrm{CH}, \mathrm{CH}^{+}, \mathrm{CN},{ }^{12} \mathrm{CO}, \mathrm{H}_{3}^{+}\right)$.

where $\sigma=0.00145$ is the standard deviation in the spectrum, $\lambda_{\text {pix }}=0.05 \AA$ is the wavelength per pixel, and $\mathcal{N}_{\text {pix }}=13$ is the number of pixels expected in a single absorption component given a $10 \mathrm{~km} \mathrm{~s}^{-1}$ FWHM (this is the average FWHM of $\mathrm{H}_{3}^{+}$ lines reported by McCall et al. 2002). These quantities result in an upper limit to the equivalent width of $W_{\lambda}<0.26 \mathrm{~m} \AA$ $\left(W_{\lambda}<0.78 \mathrm{~mA}\right.$ at the $3 \sigma$ level $)$.

Next, the standard relation between equivalent width and column density was used:

$$
N=\frac{W_{\lambda} m_{e} c^{2}}{\lambda^{2} \pi e^{2} f},
$$

where $m_{e}$ is the electron mass, $c$ is the speed of light, $\lambda$ is the wavelength of the transition, $e$ is the electron charge, and $f=0.48$ is the sum of the oscillator strengths of the two strongest, blended lines. Inserting the known parameters results in a $3 \sigma$ upper limit to the column density $N_{m}<1.6 \times 10^{9} \mathrm{~cm}^{-2}$ in a single velocity component. However, there are two cloud components along this sight line, so the total line of sight limit is $N_{m}<3.2 \times 10^{9} \mathrm{~cm}^{-2}$.

\section{ANALYSIS}

\subsection{Reaction Network Revisited}

In planning observations and calculating predicted line strengths, we relied on the simple chemical scheme that only considers the destruction of $\mathrm{He}^{+}$via electron recombination. However, during the course of this study, we identified (from advanced chemical models, e.g., Woodall et al. 2007) several competing reactions that could be important in destroying $\mathrm{He}^{+}$ in diffuse clouds:

$$
\begin{gathered}
\mathrm{He}^{+}+\mathrm{H} \rightarrow \mathrm{He}+\mathrm{H}^{+}, \\
\mathrm{He}^{+}+\mathrm{H}_{2} \rightarrow \mathrm{He}+\mathrm{H}_{2}^{+},
\end{gathered}
$$

$$
\begin{aligned}
& \mathrm{He}^{+}+\mathrm{H}_{2} \rightarrow \mathrm{He}+\mathrm{H}+\mathrm{H}^{+}, \\
& \mathrm{He}^{+}+\mathrm{CO} \rightarrow \mathrm{He}+\mathrm{O}+\mathrm{C}^{+} .
\end{aligned}
$$

Rate coefficients for these reactions, as well as the electron recombination reactions, can be determined for a specific temperature, $T$ (in Kelvin), using the fitting parameters $(\alpha, \beta$, and $\gamma$ ) in Table 2 in conjunction with the expression

$$
k=\alpha\left(\frac{T}{300}\right)^{\beta} e^{-\gamma / T} \mathrm{~cm}^{3} \mathrm{~s}^{-1} .
$$

Unlike the case of electron recombination, these reactions should not lead to metastable helium. Simple energetic arguments demonstrate why this is the case. The energy difference between the ionization potential of helium $(24.6 \mathrm{eV})$ and the excitation energy of the metastable state $(19.8 \mathrm{eV})$ is only $4.8 \mathrm{eV}$. In order to dissociate and/or ionize the reaction partners of $\mathrm{He}^{+}$, reactions (10-13) require energies of 13.6, 15.4, 18.1 , and $22.5 \mathrm{eV}$, respectively (assuming all reactants are in the ground electronic state). At diffuse cloud temperatures $(\sim 70 \mathrm{~K})$, thermal energy is much less than $1 \mathrm{eV}$, and so the kinetic energy of the reactants will have no effect. Following these arguments, the neutral helium product can only be in the ground state as more than $4.8 \mathrm{eV}$ of the helium ionization potential must be used in each reaction. As a result, accounting for these reactions greatly decreases the fraction of helium ions which pass through the triplet manifold, and thus the population of the metastable state.

However, we also made the assumption that the metastable state is only populated via cosmic-ray ionization of He, followed by electron recombination. Given that a smaller branching fraction limits this pathway, electron impact excitation into the triplet manifold will be a competing formation mechanism. Cross sections for ionization and excitation of helium by electrons in the 10-1000 eV range are shown in Figures 2a \& $2 \mathrm{~b}$ of Dalgarno et al. (1999). To compute the rate of ionization and excitation, one must perform an integral in energy space over the product of each cross section with the differential energy spectrum of electrons in the interstellar medium. This full calculation is hindered by the fact that the spectrum of secondary electrons (those produced during ionization events) is unknown, and cannot be derived from the differential energy spectrum of cosmic-ray protons which is also unknown below $\sim 1 \mathrm{GeV}$. The complexity associated with deriving the spectrum of secondary electrons is beyond the scope of this paper; thus, we make some simplifications in estimating the importance of electron impact excitation into the triplet manifold of helium.

Assuming that all secondary electrons have the same energy, the ratio between the rate of excitation into all triplet states and the rate of ionization can be determined by taking the ratio of the respective cross sections at a given energy. We take this ratio at $30 \mathrm{eV}$ (the mean value given by Cravens \& Dalgarno 1978), and find the rate of excitation into all triplet states to be 2 times faster than the rate of ionization by secondary electrons. To determine the overall importance of electron impact excitation then, we need to find a relationship between the total ionization rate of helium and the ionization rate due to secondaries. Using relations between the primary ionization rates of hydrogen and helium (Habing \& Goldsmith 1971; Liszt 2003) and between the primary ionization rate of hydrogen and the total ionization rate of helium (Glassgold \& Langer 1974; Tielens 2005), we estimate that ionization by secondary electrons accounts for about $1 / 6$ 
Table 2

Rate Coefficients for Reactions Involving Ionized Helium

\begin{tabular}{lccccc}
\hline \hline \multicolumn{1}{c}{ Reaction } & $\alpha$ & $\beta$ & $\gamma$ & $\begin{array}{c}\text { Coefficient at 70 K } \\
\left(\mathrm{cm}^{3} \mathrm{~s}^{-1}\right)\end{array}$ & $\begin{array}{c}\text { References } \\
\end{array}$ \\
\hline $\mathrm{He}^{+}+\mathrm{H} \rightarrow \mathrm{He}+\mathrm{H}^{+}$ & $1.2 \times 10^{-15}$ & 0.25 & 0 & $k_{10}=8.3 \times 10^{-16}$ & 1 \\
$\mathrm{He}^{+}+\mathrm{H}_{2} \rightarrow \mathrm{He}+\mathrm{H}_{2}^{+}$ & $7.2 \times 10^{-15}$ & 0 & 0 & $k_{11}=7.2 \times 10^{-15}$ & 2 \\
$\mathrm{He}^{+}+\mathrm{H}_{2} \rightarrow \mathrm{He}+\mathrm{H}+\mathrm{H}^{+}$ & $3.7 \times 10^{-15}$ & 0 & 35 & $k_{12}=2.2 \times 10^{-14}$ & 2 \\
$\mathrm{He}^{+}+\mathrm{CO} \rightarrow \mathrm{He}+\mathrm{O}+\mathrm{C}^{+}$ & $1.6 \times 10^{-9}$ & 0 & 0 & $k_{13}=1.6 \times 10^{-9}$ & 3,4 \\
$\mathrm{He}^{+}+e \rightarrow \mathrm{He}\left(1^{1} \mathrm{~S}\right)+h v$ & $1.76 \times 10^{-12}$ & -0.56 & 0 & $\alpha_{1}=4.0 \times 10^{-12}$ & 5 \\
$\mathrm{He}^{+}+e \rightarrow \mathrm{He}\left(2^{3} \mathrm{~S}\right)+h v$ & $2.84 \times 10^{-12}$ & -0.59 & 0 & $\alpha_{3}=6.6 \times 10^{-12}$ & 5 \\
\hline
\end{tabular}

Notes. Coefficients at temperatures between about 10 and $300 \mathrm{~K}$ can be derived using $\alpha, \beta, \gamma$, and Equation (14). Rate coefficients and their references for reactions (10)-(13) were found at http://www.udfa.net/.

References (1) Stancil et al. 1998; (2) Barlow 1984; (3) Laudenslager et al. 1974; (4) Anicich et al. 1977 and (5) R. Porter 2009, private communication.

of the total ionization rate of helium. This, in turn, leads to the approximation that the rate for electron impact excitation into the triplet manifold - and thus the metastable state (which we denote $\delta_{\mathrm{He}^{*}}$ ) - should be roughly $1 / 3$ that of the total ionization rate of helium (i.e., $\delta_{\mathrm{He}^{*}} \approx \zeta_{\mathrm{He}} / 3$; we use this relation for the remainder of this paper).

Mathematically, these additional formation and destruction reactions can easily be included by altering the steady state equations in Section 1.2, resulting in two changes to our analysis. First, due to the additional destruction pathways of $\mathrm{He}^{+}$, the branching fraction, $b$, must be redefined as

$$
b \equiv \frac{\alpha_{3} n_{e}}{n(\mathrm{H}) k_{10}+n\left(\mathrm{H}_{2}\right)\left(k_{11}+k_{12}\right)+n(\mathrm{CO}) k_{13}+n_{e}\left(\alpha_{1}+\alpha_{3}\right)} .
$$

In many cases, however, absolute abundances are not known, and it is thus convenient to recast Equation (15) in terms of fractional abundances as

$$
b=\frac{\alpha_{3} x_{e}}{\left(1-f_{\mathrm{H}_{2}}\right) k_{10}+f_{\mathrm{H}_{2}}\left(k_{11}+k_{12}\right) / 2+x_{\mathrm{CO}} k_{13}+x_{e}\left(\alpha_{1}+\alpha_{3}\right)},
$$

where $x_{j} \equiv n_{j} / n_{\mathrm{H}}, n_{\mathrm{H}} \equiv n(\mathrm{H})+2 n\left(\mathrm{H}_{2}\right)$, and the molecular hydrogen fraction $f_{\mathrm{H}_{2}} \equiv 2 n\left(\mathrm{H}_{2}\right) / n_{\mathrm{H}}$. Second, Equation (6) must be recast to include electron impact excitation into the metastable state, and becomes

$$
\frac{1}{f_{m}} \approx \frac{A}{b \zeta_{\mathrm{He}}+\delta_{\mathrm{He}^{*}}} .
$$

While the analysis now includes many more parameters, we can still calculate the fractional abundance of metastable helium, and thus the expected line strength, toward HD 183143. We assume that fractional abundances are constant throughout the cloud, allowing us to substitute column densities for number densities when available (i.e., $x_{j}=N_{j} / N_{\mathrm{H}}$ ). Using the color excess as in Section 2.1 gives $N_{\mathrm{H}}=7.4 \times 10^{21} \mathrm{~cm}^{-2}$. This is used in conjunction with spectroscopic observations of $\mathrm{CO}$ which indicate $N(\mathrm{CO}) \approx 10^{15} \mathrm{~cm}^{-2}$ (McCall et al. 2002) to compute $x_{\mathrm{CO}}$. The assumption that there are equal amounts of atomic and molecular hydrogen is quantified by $f_{\mathrm{H}_{2}}=2 / 3$. Finally, observations of $\mathrm{C}^{+}$in diffuse clouds have shown that $x_{e} \sim 1.4 \times 10^{-4}$, assuming that nearly all electrons come from this singly ionized carbon (Cardelli et al. 1996). Combining these data and assumptions with the rate coefficients in Table 2, the new branching fraction is $b=0.08$, about one-eighth of the value considering electrons alone. Substituting this branching fraction and the relevant parameters from Sections 2.1 and 2.2 into Equation (17) results in values of $f_{m}=1.3 \times 10^{-12}$,
$N_{m}=9.3 \times 10^{8} \mathrm{~cm}^{-2}$, and $W_{\lambda}=0.46 \mathrm{~m} \AA$. Again splitting the material into two equal cloud components decreases the equivalent widths to $W_{\lambda}=0.23 \mathrm{~m} \AA$, which would require a $\mathrm{S} / \mathrm{N} \sim 2000$ for a $3 \sigma$ detection.

\subsection{Cosmic-ray Ionization Rate of Helium}

Re-arranging Equation (17), we can turn this problem around and compute an upper limit to the cosmic-ray ionization rate of helium using our observations. Given the upper limit to the metastable column density, $N_{m}<3.2 \times 10^{9} \mathrm{~cm}^{-2}$, and the estimated total helium column, $N(\mathrm{He})=\beta A(\mathrm{He}) E(B-V)=$ $7.1 \times 10^{20} \mathrm{~cm}^{-2}$, the $3 \sigma$ upper limit to the fractional metastable population is $f_{m}<4.5 \times 10^{-12}$. Using this in concert with the branching fraction above, $b=0.08$, results in $\zeta_{\mathrm{He}}<1.2 \times 10^{-15}$ $\mathrm{s}^{-1}$. This upper limit is about 5 times larger than the ionization rate inferred from $\mathrm{H}_{3}^{+}$observations (assuming the relation between the ionization rate of helium and molecular hydrogen is given by $2.3 \zeta_{\mathrm{He}}=1.5 \zeta_{2}$; Glassgold \& Langer 1974). Because of electron impact excitation into the metastable state though, this determination of the ionization rate relies on a much more indirect analysis than was initially proposed. Comparing $b \zeta_{\mathrm{He}}$ to $\delta_{\mathrm{He}^{*}}$, we can compute the relative importance of each formation mechanism via

$$
P\left(\delta_{\mathrm{He}^{*}}\right)=\frac{\delta_{\mathrm{He}^{*}}}{b \zeta_{\mathrm{He}}+\delta_{\mathrm{He}^{*}}}=(3 b+1)^{-1} .
$$

In doing so, we find that electron impact excitation accounts for $80 \%$ of the metastable population, while ionization and electron recombination accounts for $20 \%$.

\section{DISCUSSION}

While the reactions associated with metastable helium are more complex than previously presented, we still see it as a viable tracer of the cosmic-ray ionization rate. As such, we decided to investigate the prospects for $\mathrm{He}^{*}$ detections in various interstellar environments, including diffuse molecular clouds $\left(100 \mathrm{~cm}^{-3} \lesssim n_{\mathrm{H}} \lesssim 500 \mathrm{~cm}^{-3}, f_{\mathrm{H}_{2}} \gtrsim 0.1\right)$, dense clouds $\left(n_{\mathrm{H}} \gtrsim\right.$ $\left.10^{4} \mathrm{~cm}^{-3}, \widetilde{f_{\mathrm{H}_{2}}} \approx 1\right)$, and diffuse atomic clouds $\left(n_{\mathrm{H}} \lesssim 100 \mathrm{~cm}^{-\widetilde{3}}\right.$, $f_{\mathrm{H}_{2}} \lesssim 0.1$; Snow \& McCall 2006). The following analyses will highlight the branching fraction in each environment, as well as the relative importance of electron impact excitation versus ionization + recombination using Equation (18).

\subsection{Diffuse Molecular Clouds}

Given that the analysis in Section 2.1 did not account for the processes examined in Section 5.1, we felt it prudent to 
revisit the calculations for diffuse molecular clouds. We use the same values as before $\left(E(B-V)=1 ; \zeta_{\mathrm{He}}=3 \times 10^{-16}\right.$ $\mathrm{s}^{-1}$ ), but now also assume $f_{\mathrm{H}_{2}}=2 / 3, x_{e}=1.4 \times 10^{-4}$, and $x_{\mathrm{CO}}=10^{-7}$. The general results for this environment $(b=0.08$; $\left.f_{m}=1.1 \times 10^{-12} ; N_{m}=6.3 \times 10^{8} \mathrm{~cm}^{-2} ; W_{\lambda}=0.31 \mathrm{~m} \AA\right)$ are similar to those for the specific diffuse molecular sight line HD 183143, with the differences due to the lower color excess. Assuming that all of the material has the same velocity, metastable helium absorption should be observable in diffuse molecular clouds at a $3 \sigma$ level with $\mathrm{S} / \mathrm{N} \sim 1500$. Given the small branching fraction above, $P\left(\delta_{\mathrm{He}^{*}}\right)=0.8$, and we conclude that metastable helium is predominantly formed via electron impact excitation in diffuse molecular clouds.

\subsection{Dense Clouds}

Dense clouds, while providing a larger total helium column, have several characteristics detrimental to the formation of metastable helium. The cosmic-ray ionization rate tends to be about one order of magnitude lower in dense clouds than in diffuse clouds (Dalgarno 2006). Also, the fractional abundance of electrons is much lower, $x_{e} \approx 4 \times 10^{-8}$, while the fractional abundance of $\mathrm{CO}$ is much higher, $x_{\mathrm{CO}} \approx 1.4 \times 10^{-4}$ (Woodall et al. 2007). Because $k_{13}$ is so much larger than any of the other rate coefficients, collisions with $\mathrm{CO}$ will dominate the destruction of $\mathrm{He}^{+}$and Equation (16) can be simplified to

$$
b \approx \frac{x_{e} \alpha_{3}}{x_{\mathrm{CO}} k_{\mathrm{CO}}} .
$$

Given the fractional abundances above and the relevant rate coefficients $\left(\alpha_{3}\right.$ and $k_{\mathrm{CO}}$ were computed for $\left.T=40 \mathrm{~K}\right)$, the branching fraction is $b \sim 10^{-6}$. As a result, $P\left(\delta_{\mathrm{He}^{*}}\right) \approx 1$, meaning that metastable helium is formed exclusively by electron impact excitation in dense clouds. Even with this formation mechanism though, the expected equivalent width $\left(W_{\lambda}=0.13 \mathrm{~m} \AA\right)$ and necessary $\mathrm{S} / \mathrm{N}$ for a $3 \sigma$ detection $(\mathrm{S} / \mathrm{N} \sim 3700)$, coupled with the large attenuation of the background star's flux at $1 \mu \mathrm{m}$, make the detection of He* in dense clouds highly unlikely.

\subsection{Diffuse Atomic Clouds}

Diffuse atomic clouds, on the other hand, have negligible concentrations of $\mathrm{H}_{2}$ and $\mathrm{CO}$ (Snow \& McCall 2006) and presumably share the high ionization rate of diffuse molecular clouds. In purely atomic conditions, electron recombination only has to compete with reaction (10), and Equation (16) can be approximated as

$$
b \approx \frac{x_{e} \alpha_{3}}{k_{10}+x_{e}\left(\alpha_{1}+\alpha_{3}\right)} .
$$

The simplified result for atomic clouds is then $b \approx 0.40$, with a corresponding $P\left(\delta_{\mathrm{He}^{*}}\right)=0.45$, meaning that ionization and electron impact excitation play roughly equal roles in forming metastable helium in such environments. Despite this branching fraction being closer to the ideal case of $b=0.62$, the low amount of material along such a sight line $(E(B-$ $V) \sim 0.1)$ results in a predicted equivalent width of $W_{\lambda} \approx$ $0.06 \mathrm{~m} \AA$. However, there are some diffuse atomic sight lines with more favorable conditions. One such candidate, $\sigma$ Sco, has $E(B-V)=0.40$ (Clayton \& Hanson 1993) and thus a predicted equivalent width of $W_{\lambda} \approx 0.22 \mathrm{~m} \AA$ using Equations (17) and (20). However, $\sigma$ Sco also has measured values of $N(\mathrm{H})=$
$2.4 \times 10^{21} \mathrm{~cm}^{-2}, N\left(\mathrm{H}_{2}\right)=6.2 \times 10^{19} \mathrm{~cm}^{-2}$ (Savage et al. 1977), and $N(\mathrm{CO})=6.5 \times 10^{12} \mathrm{~cm}^{-2}$ (Allen et al. 1990), which correspond to $f_{\mathrm{H}_{2}}=0.049$ and $x_{\mathrm{CO}}=2.6 \times 10^{-9}$. Using these values and Equation (16), we can test the accuracy of Equation (20) at small molecular fractions. The result is $b=0.31$, or about a $30 \%$ error in the approximation. At $f_{\mathrm{H}_{2}}=0.15$, Equation (20) overestimates $b$ by a factor of 2 , so this approximation should only be applied for $f_{\mathrm{H}_{2}} \lesssim 0.1$. Taking the branching fraction from the full calculation, we predict an equivalent width of $W_{\lambda} \approx 0.20 \mathrm{~m} \AA$, and a corresponding $\mathrm{S} / \mathrm{N} \sim 2400$ necessary for a $3 \sigma$ detection. If such a detection can be made, however, it will provide the exciting opportunity to probe the cosmic-ray ionization rate in an environment where $\mathrm{H}_{3}^{+}$observations cannot be made due to the low molecular fraction.

\section{CONCLUSIONS}

We have analyzed the possibility of detecting absorption lines due to interstellar metastable helium at $10830 \AA$ A. Observations toward the diffuse cloud sight line HD 183143 were taken, and a spectrum with $\mathrm{S} / \mathrm{N} \sim 700$ was obtained, but no interstellar He I* lines were detected. In examining the chemistry associated with metastable helium, we have identified important formation and destruction pathways, and have derived new equations for the steady state analysis of $\mathrm{He}^{*}$. While these reactions have been known for some time, this is the first instance where they have been applied to metastable helium chemistry. Using our observations and the newly derived equations, we inferred an upper limit for the cosmic-ray ionization rate of helium which, although consistent with other studies, is about 5 times larger than previously inferred values.

To determine if future observations of interstellar $\mathrm{He}^{*}$ are warranted, we predicted the S/N ratios necessary for $3 \sigma$ detections in various environments. Diffuse molecular clouds are the most promising targets with $\mathrm{S} / \mathrm{N} \sim 1500$ required, while favorable diffuse atomic clouds need $\mathrm{S} / \mathrm{N} \sim 2400$. While such observations are extremely challenging at present, advancements in telescope and near-infrared detector technology may one day make metastable helium a widely applicable probe of the cosmic-ray ionization rate. In diffuse molecular clouds, $\mathrm{He}^{*}$ will act as a cosmic-ray probe independent of $\mathrm{H}_{3}^{+}$, and together with $\mathrm{H}_{3}^{+}$it will also enable determination of the absorption path length and average cloud density. He* observations will also be especially important for diffuse atomic clouds, where there are no other reliable tracers of the ionization rate.

The authors wish to thank Tom Kerr and the UKIRT Service Observing Programme for obtaining preliminary spectra near $10830 \AA$ using CGS4, Ryan Porter and Gary Ferland for supplying unpublished values of the recombination coefficients and for suggesting the possibility of electron impact excitation into the metastable state, and the anonymous referee for helpful suggestions and comments. N.I. and B.J.M. have been supported by NSF grant PHY 05-55486.

Based on observations obtained at the Gemini Observatory, which is operated by the Association of Universities for Research in Astronomy, Inc., under a cooperative agreement with the NSF on behalf of the Gemini partnership: the National Science Foundation (United States), the Science and Technology Facilities Council (United Kingdom), the National Research Council (Canada), CONICYT (Chile), the Australian Research Council (Australia), Ministério da Ciência e Tecnologia (Brazil), and Ministerio de Ciencia, Tecnología e Innovación Produc- 
tiva (Argentina). The Gemini/Phoenix spectra were obtained through program GS-2008A-Q-14.

The observations were obtained with the Phoenix infrared spectrograph, which was developed by the National Optical Astronomy Observatory.

Note added in proof. We would like to thank Nick Abel for bringing to our attention the reaction $\mathrm{He}^{+}+\mathrm{H} \rightarrow \mathrm{HeH}^{+}+h v$, which acts in a similar manner as reactions (10)-(13). The rate coefficient for this reaction at about $70 \mathrm{~K}$ is $k \sim 2 \times 10^{-15} \mathrm{~s}^{-1}$ (Roberge \& Dalgarno 1982; Kraemer et al. 1995). Adding this reaction to our analysis has a negligible effect on the dense cloud and diffuse molecular cloud results, but changes the results for the diffuse atomic cloud $\sigma$ Sco as follows: $b=0.19$, $P\left(\delta_{\mathrm{He}^{*}}\right)=0.64, W_{\lambda} \approx 0.16 \mathrm{~m} \AA$, and $\mathrm{S} / \mathrm{N} \sim 3000$.

\section{REFERENCES}

Ádámkovics, M., Blake, G. A., \& McCall, B. J. 2003, ApJ, 595, 235 Allen, M. M., Snow, T. P., \& Jenkins, E. B. 1990, ApJ, 355, 130

Anders, E., \& Grevesse, N. 1989, Geochim. Cosmochim. Acta, 53, 197

Anicich, V. G., Laudenslager, J. B., Huntress, W. T., \& Futrell, J. H. 1977, J. Chem. Phys., 67, 4340

Barlow, S. E. 1984, PhD thesis, Univ. of Colorado

Black, J. H., \& Dalgarno, A. 1977, ApJS, 34, 405

Black, J. H., Hartquist, T. W., \& Dalgarno, A. 1978, ApJ, 224, 448

Bohlin, R. C., Savage, B. D., \& Drake, J. F. 1978, ApJ, 224, 132

Cardelli, J. A., Meyer, D. M., Jura, M., \& Savage, B. D. 1996, ApJ, 467, 334

Clayton, G. C., \& Hanson, M. M. 1993, AJ, 105, 1880

Cravens, T. E., \& Dalgarno, A. 1978, ApJ, 219, 750

Dalgarno, A. 2006, Proc. Natl Acad. Sci., 103, 12269

Dalgarno, A., Yan, M., \& Liu, W. 1999, ApJS, 125, 237

Drake, G. W. F., \& Dalgarno, A. 1968, ApJ, 152, L121

Federman, S. R., Weber, J., \& Lambert, D. L. 1996, ApJ, 463, 181
Glassgold, A. E., \& Langer, W. D. 1974, ApJ, 193, 73

Gredel, R., van Dishoeck, E. F., \& Black, J. H. 1993, A\&A, 269, 477

Griem, H. R. 1969, ApJ, 156, L103

Habing, H. J., \& Goldsmith, D. W. 1971, ApJ, 166, 525

Hartquist, T. W., Black, J. H., \& Dalgarno, A. 1978a, MNRAS, 185, 643

Hartquist, T. W., Doyle, H. T., \& Dalgarno, A. 1978b, A\&A, 68, 65

Hata, J., \& Grant, I. P. 1981, J. Phys. B: At. Mol. Phys., 14, 2111

Hinkle, K., et al. 2002, Proc. SPIE, 4834, 353

Indriolo, N., Geballe, T. R., Oka, T., \& McCall, B. J. 2007, ApJ, 671, 1736

Kraemer, W. P., Špirko, V., \& Juřek, M. 1995, Chem. Phys. Lett., 236, 177

Kulesa, C. A. 2002, PhD thesis, Univ. Arizona

Laudenslager, J. B., Huntress, W. T., \& Bowers, M. T. 1974, J. Chem. Phys., 61,4600

Liszt, H. 2003, A\&A, 398, 621

McCall, B. J. 2001, PhD thesis, Univ. of Chicago

McCall, B. J., et al. 2002, ApJ, 567, 391

McCall, B. J., et al. 2003, Nature, 422, 500

Le Petit, F., Roueff, E., \& Herbst, E. 2004, A\&A, 417, 993

O’Donnell, E. J., \& Watson, W. D. 1974, ApJ, 191, 89

Ralchenko, Y., Kramida, A. E., Reader, J., \& NIST ASD Team 2008, NIST Atomic Spectra Database (version 3.1.5; Gaithersburg, MD: National Institute of Standards and Technology), http://physics.nist.gov/asd3

Rees, M. J., Sciama, D. W., \& Stobbs, S. H. 1968, Astrophys. Lett., 2, 243

Roberge, W., \& Dalgarno, A. 1982, ApJ, 255, 489

Savage, B. D., Bohlin, R. C., Drake, J. F., \& Budich, W. 1977, ApJ, 216, 291

Scherb, F. 1968, ApJ, 153, L55

Snow, T. P., \& McCall, B. J. 2006, ARA\&A, 44, 367

Spitzer, L., Jr, \& Tomasko, M. G. 1968, ApJ, 152, 971

Stancil, P. C., Lepp, S., \& Dalgarno, A. 1998, ApJ, 509, 1

Thorburn, J. A., et al. 2003, ApJ, 584, 339

Tielens, A. G. G. M. 2005, The Physics and Chemistry of the Interstellar Medium (Cambridge: Cambridge Univ. Press)

van Dishoeck, E. F., \& Black, J. H. 1986, ApJS, 62, 109

Webber, W. R. 1998, ApJ, 506, 329

Woodall, J., Agúndez, M., Markwick-Kemper, A. J., \& Millar, T. J. 2007, A\&A, 466, 1197

Woodworth, J. R., \& Moos, H. W. 1975, Phys. Rev. A, 12, 2455 